\title{
Inconsistency Scale
}

National Cancer Institute

\section{Source}

National Cancer Institute. Inconsistency Scale. NCI Thesaurus. Code C159491.

Uses the 10 item pairs. For each pair the absolute value of the difference for the items is summed to get the Inconsistency Scale score. 